\title{
Time-scale invariances in preseismic electromagnetic radiation, magnetization and damage evolution of rocks
}

\author{
Y. Kawada ${ }^{1,2}$, H. Nagahama ${ }^{1}$, and N. Nakamura ${ }^{1}$ \\ ${ }^{1}$ Department of Geoenvironmental Sciences, Graduate School of Science, Tohoku University, Sendai, Japan \\ ${ }^{2}$ School of GeoSciences, University of Edinburgh, Edinburgh, UK
}

Received: 23 July 2007 - Revised: 9 October 2007 - Accepted: 9 October 2007 - Published: 19 October 2007

\begin{abstract}
We investigate the time-scale invariant changes in electromagnetic and mechanical energy releases prior to a rock failure or a large earthquake. The energy release processes are caused by damage evolutions such as crack propagation, motion of charged dislocation, area-enlargement of sheared asperities and repetitive creep-rate changes. Damage mechanics can be used to represent the time-scale invariant evolutions of both brittle and plastic damages. Irreversible thermodynamics applied to the damage mechanics reveals that the damage evolution produces the variations in charge, dipole and electromagnetic signals in addition to mechanical energy release, and yields the time-scale invariant patterns of Benioff electromagnetic radiation and cumulative Benioff strain-release. The irreversible thermodynamic framework of damage mechanics is also applicable to the seismo-magnetic effect, and the time-scale invariance is recognized in the remanent magnetization change associated with damage evolution prior to a rock failure.
\end{abstract}

\section{Introduction}

Prior to a large earthquake or a rock failure, a critical phenomenon of electromagnetic energy release has often been observed and termed the "Benioff electromagnetic radiation" (e.g., Rabinovitch et al., 2001; Eftaxias et al., 2004, 2007; Kapiris et al., 2004a, b). This is quantified by the sum of the earthquake amplitudes and displays a time-scale invariance. A similar phenomenon has also known as the timescale invariant pattern of mechanical energy release quantified by cumulative Benioff strain-release (: the sum of square roots of released energy from the respective seismic events) (e.g., Varnes, 1989; Bufe and Varnes, 1993; Bowman et al., 1998). Seismo-electromagnetic phenomena are gener-

Correspondence to: Y. Kawada

(y-kawada@mail.tains.tohoku.ac.jp) ally due directly or indirectly to mechanical processes such as the piezoelectric effect (e.g., Finkelstein et al., 1973), the electrokinetic effect (e.g, Mizutani et al., 1976), point defects (e.g., Varotsos and Alexopoulos, 1986), opening and propagation of charged cracks (e.g., Ogawa et al., 1985; O'Keefe and Thiel, 1995; Vallianatos and Tzanis, 1999; Tzanis et al., 2000; Takeuchi and Nagahama, 2001, 2002a, b, 2004, 2006; Tzanis and Vallianatos, 2002; Vallianatos et al., 2004), and motion of charged dislocations (e.g., Gokhberg et al., 1995; Molchanov and Hayakawa, 1995; Nagahama and Teisseyre, 1998; Freund, 2000; Teisseyre and Nagahama, 1999, 2001; Tzanis and Vallianatos, 2002; Vallianatos et al., 2004; Triantis et al., 2006). However, the relationship between electromagnetic and mechanical energy release prior to an earthquake and rock failure has not previously been studied in terms of its time-scale invariance.

The statistical properties of cumulative Benioff strainrelease have been investigated using the irreversible thermodynamic models such as continuum damage and fibrebundle models, revealing time-scale invariant evolutions of damage (e.g., Main, 1999; Rundle et al., 2000; Ben-Zion and Lyakhovsky, 2002, Turcotte et al., 2003; Kawada and Nagahama, 2006). Several types of damage evolution such as crack opening, crack propagation, motion of charged dislocation and area-enlargement of sheared asperity are origins of emitted electromagnetic signals. In particular, Vallianatos et al. (2004), Triantis et al. (2006) and Anastasiadis et al. (2007) discussed the pressure stimulated currents due to the damage evolution based on the irreversible thermodynamic model (continuum damage model, Turcotte et al., 2003) and statistical mechanical model of crack population (e.g., Czechowski, 1991, 1995; Tzanis et al., 2000; Tzanis and Vallianatos, 2002; Vallianatos et al., 2004). Moreover, Muto et al. (2007) pointed out that the rate of frictional discharge (plasma generation, i.e., ionization current) depends on the temporal evolution of the area density of fault asperities. When the current is related to the intensity of inducted

Published by Copernicus Publications on behalf of the European Geosciences Union. 


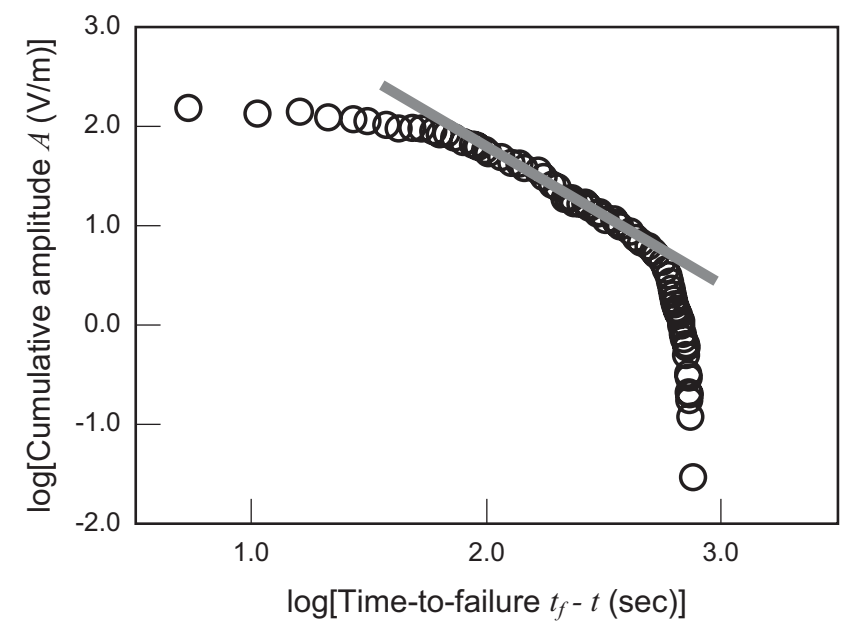

Fig. 1. The log-log plots of the time-to-failure and cumulative amplitude $A$ of signals registered during chalk samples compression. This figure is adapted from Rabinovitch et al. (2001).

electromagnetic field, the damage evolution is linked to the Benioff electromagnetic radiation. However, the damage evolution has not been coupled with the charge generation mechanism and the Benioff electromagnetic radiation via irreversible thermodynamics including time-scale invariance.

By contrast, the seismo-magnetic effect (e.g., rock magnetization) is also expected prior to a seismic event or rock failure, although the seismo-magnetic effect is not always beyond the stationary level of the geomagnetic effects (e.g., Rikitake, 1976). Traditionally, the seismo-magnetic effects are investigated in terms of the elastic deformation (e.g., Kern, 1961; Stacey, 1964; Sasai, 1980, 1991, 1994). Nakamura and Nagahama (1997a, b, 1999, 2001) and Muto and Nagahama (2004) extended the seismo-magnetic theory and explained rock magnetization associated with plastic deformation. However, the magnetization associated with timescale invariant evolution of damage has yet to be addressed.

In this paper, we investigate the temporal change in electromagnetic radiation associated with damage evolution prior to rock failure or seismic events. First, we review the observational and experimental results in electromagnetic radiation and the fundamentals of damage mechanics. We then clarify how damage evolution affects Benioff electromagnetic radiation, and couple the time-scale invariant changes in elastic and electromagnetic energy release using the irreversible thermodynamics for damage evolution. In addition, based on the irreversible framework, we extend the theory on seismo-magnetic effects associated with damage evolution prior to rock failure and seismic events.

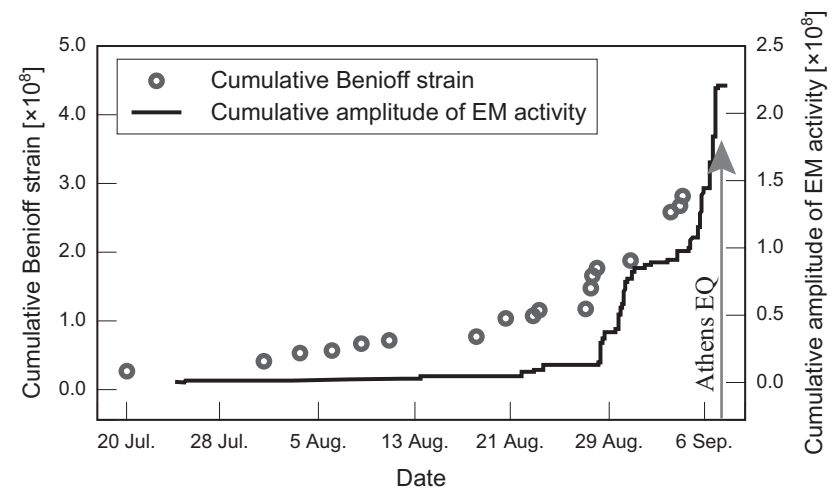

Fig. 2. The time-series of cumulative Benioff strain and cumulative amplitude of electromagnetic (EM) activity (i.e., Benioff electromagnetic radiation) prior to the 1999 Athens earthquake (occurred at 7 September $1999 ; 38.15^{\circ} \mathrm{N}, 23.62^{\circ} \mathrm{E}$ ). The analyzed data of EM activities is $10 \mathrm{kHz}$; time-series monitored at Zenta station in western Greece $\left(37.76^{\circ} \mathrm{N}, 20.76^{\circ} \mathrm{E}\right)$. The seismic data is from the catalogue of earthquakes (local magnitude $M \geq 2.8$ ) with hypocentres $\leq 60 \mathrm{~km}$, located within $110 \mathrm{~km}$ from the hypocentre of the Athens earthquake. This figure is modified from Kapiris et al. (2004a).

\section{Benioff electromagnetic radiation}

In this section, we present on an overview the results of laboratory experiments and observations on the Benioff electromagnetic radiation. Rabinovitch et al. (2001) conducted both uniaxial and triaxial compression tests of 24 chalk samples, and monitored amplitude changes as electromagnetic radiation associated with cracking and fracturing. The amplitude changes were measured by a one-loop magnetic antenna in the frequency band from $50 \mathrm{kHz}$ to $10 \mathrm{MHz}$ (see also Rabinovitch et al., 1998). The measured amplitude is proportional to magnetic field intensity and to the square root of electromagnetic energy $\sqrt{e_{L}}$. Rabinovitch et al. (2001) analyzed the relationship between time-to-failure $t_{f}-t$ and the cumulative amplitude $A$ of all signals registered during all samples' compression (Fig. 1), and found a power-law relation of the Benioff electromagnetic radiation,

$A=A_{0}-A_{1}\left(t_{f}-t\right)^{\gamma_{A}}$,

where $\gamma_{A}$ is an exponent, and $A_{0}$ and $A_{1}$ are constants (Fig. 1).

Eftaxias et al. (2004, 2006, 2007) and Kapiris et al. (2004a, b) reported the Benioff electromagnetic radiation prior to the 1999 Athens earthquake. They analyzed the amplitudes of kilohertz electromagnetic activities during 10 May 1999-7 September 1999, and showed that the Benioff electromagnetic radiation follows Eq. (1) with $t_{f}$ being 7 September, 1999 and $A$ being the sum of the amplitudes from 10 May, 1999 (Fig. 2). Moreover, they analyzed the temporal seismicity pattern of cumulative Benioff strain-release $\Omega$ using the 
catalogue of earthquakes (local magnitude $M \geq 2.8$ ) during 10 May 1999 to 7 September 1999, and derived the equation,

$$
\left(\sum \sqrt{e_{M}} \equiv\right) \Omega=\Omega_{0}-\Omega_{1}\left(t_{f}-t\right)^{-\gamma_{\Omega}},
$$

where $e_{M}$ is the mechanical energy released at each seismic event, $\gamma_{\Omega}$ is an exponent, and $\Omega_{0}$ and $\Omega_{1}$ are constants (see also Fig. 2, and Tzanis and Makropoulos, 2002). Thus, the temporal patterns of electromagnetic and mechanical energy release were both time-scale invariant. Nanjo and Nagahama $(2004 a, b)$ showed that the elastic energy from the force locally lost is converted into the electromagnetic radiation when the elastic-force redistribution of earthquakes is locally non-conservative. As noted earlier, the cumulative Benioff strain-release is known to be due to time-scale invariant evolution of damage (e.g., Kawada and Nagahama, 2006). In the next section, we review the cumulative Benioff strain-release in terms of irreversible thermodynamic models according to Kawada and Nagahama (2006) and Kawada et al. (2007).

\section{Damage evolution and cumulative Benioff strain- release}

The irreversible thermodynamic model for damage evolution enables us to represent the observable elastic or viscoelastic behaviour associated with microscopic irreversible processes. Fibre-bundle models (e.g., Turcotte et al., 2003; Sornette, 2003; Nanjo and Turcotte, 2005; Kawada and Nagahama, 2006) and continuum damage models (e.g., Lyakhovsky et al., 1993, 1997; Ben-Zion and Lyakhovsky, 2002; Turcotte et al., 2003) are often used to investigate the brittle behaviour of rocks.

In irreversible thermodynamics models, free energy is defined as a function of various state variables, including "internal state variables" (or "hidden variables") not available for macroscopic observation (e.g., Biot, 1954; Schapery, 1964; Lyakhovsky et al., 1993, 1997; Kawada et al., 2006). The internal state variables are often represented by the generalized coordinates. To investigate the damage evolution, a damage parameter $\alpha$ is introduced into the internal state variable. In brittle damage processes, we define $\alpha$ as a density of microcracks in a laboratory specimen, or as an area density of asperities in a fault plane or a contact surface of a rock sample, with $0<\alpha<1$. At $\alpha=0$, the material is undamaged, while it fails at a critical value $\alpha_{c} . \alpha$ is also regarded as porosity in soil or sediment deformation, and as a density of line vacancies or dislocations in a plastic process.

The viscoelastic behaviour of rocks associated with or without damage evolution is empirically expressed by Dorn's equation $\dot{\varepsilon} \propto \sigma^{\rho}$ (e.g. Lankford, 1981; Poirier, 1985; Nanjo and Turcotte, 2005). This power-law relationship is changeable into a time-scale invariant decay of relaxation;

$\frac{d \sigma}{d \varepsilon} \equiv E(t) \propto t^{-1 / \rho}$, where $\sigma$ and $\varepsilon$ are the macroscopic elastic stress and strain, respectively, $E(t)$ is the relaxation modulus, and $\rho$ ranges from 1 to 60 (e.g. Kawada and Nagahama, 2004, 2006; Kawada et al., 2006). $\rho$ reflects the deformation mechanisms of brittle and viscoelastic behaviour (e.g., Nakamura and Nagahama, 1999). Importantly, the stress-strain relation associated with damage evolution is based on irreversible thermodynamics (Schapery, 1964, 1969; Kawada et al., 2006) by

$\sigma=h_{e} \frac{d \alpha}{d \varepsilon} \int E\left(t-t^{\prime}\right) \frac{d \varepsilon}{d t^{\prime}} d t^{\prime}$,

where $t^{\prime}$ is the arbitrary deformation time, and $h_{e}$ is a constant. Note that $\alpha$ contributes towards the irreversible (brittle or plastic) component of the deformation. Equations (3) and (4) form the constitutive equation of viscoelastic behaviour with damage evolution.

The continuum damage and fibre-bundle models recover the microscopic damage evolution $d \alpha / d t$,

$\frac{d \alpha}{d t} \propto\left(t_{c}-t\right)^{\frac{2-\rho}{\rho-1}}$,

where $t_{c}$ is the time of failure (e.g. Lyakhovsky et al., 1993, 1997; Turcotte et al., 2003; Kawada and Nagahama, 2006). The time-scale invariance in Eq. (5) is based on the stochastic process of damage evolution following the Weibull distribution (e.g., Turcotte et al., 2003, Nanjo and Turcotte, 2005). Hence Eq. (5) is related to the special case of Czechowski's model of crack population (e.g., Czechowski, 1991, 1995; Tzanis and Vallianatos, 2002, Vallianatos et al., 2004). It can be shown that the fibre-bundle model also recovers the cumulative Benioff strain-release, since

$\Omega=\Omega_{0}^{\prime}-\Omega_{1}^{\prime}\left(t_{c}-t\right)^{\frac{\rho-2}{2(\rho-1)}}$,

where $\Omega_{0}^{\prime}$ and $\Omega_{1}^{\prime}$ are constants (e.g. Ben-Zion and Lyakhovsky, 2002; Turcotte et al., 2003; Kawada and Nagahama, 2006). Comparing Eqs. (2) and (6), $t_{f}$ corresponds to $t_{c}$ and $\gamma_{\Omega}=(\rho-2) /[2(\rho-1)]$. Hence the temporal evolution of $\Omega$ is regulated by the time-scale invariance in damage evolution and viscoelastic behaviour. A similar scenario to Eq. (6) also emerges in the time-series of atmospheric radon concentration prior to a large earthquake (e.g., Yasuoka et al., 2006). Kawada et al. (2007) pointed out that the damage evolution regulated by Eq. (5) promotes radon migration within crustal rocks, and migration affects the time-scale invariant increase in the flux of radon into the atmosphere. Furthermore, the invariances in cumulative Benioff strain-release are found to be related to the fractal structure of the pre-existing fault system (Nanjo and Nagahama, 2004a, b).

\section{Generation of charge and dipole due to damage evo- lution}

Let us consider a mechanism generating charge and a dipole due to the brittle damage evolution, that is, the charge separation on a pair of crack walls (e.g., Ogawa et al., 1985; 
O'Keefe and Thiel, 1995; Tzanis and Vallianatos, 2002; Takeuchi and Nagahama, 2004, 2006). The surface charge $Q$ on the crack walls increases with crack propagation, and the pair of crack walls generates an electric dipole $p=Q l$, where $l$ is the separation distance. The charges and dipoles rapidly decay to zero when the crack propagations ceases.

A fault plane or contact surface of rock sample in a stickslip experiment is supported by many asperities. Shearing the asperities generates and propagates the cracks, and the asperities finally fail. The failure of one asperity corresponds to a seismic event or stick-slip event. Hence the area-enlargement of sheared asperities increases the number of electric dipoles in the respective cracks (e.g., Takeuchi and Nagahama, 2004, 2006; Muto et al., 2007). When the fault plane or contact area of the sample is approximately flat, the area-enlargement of sheared asperities regulates the rate of summation of the dipoles in the respective cracks $\frac{d}{d t}\left(\sum p\right) \equiv \frac{d}{d t} P$. Note that the temporal variation in total electric dipole concentration is related to the change in the intensity of the electromagnetic field. Hence when the area density of sheared asperities is regarded as $\alpha$ in Eq. (5) (e.g., Kawada and Nagahama, 2006; Muto et al., 2007), the summation of dipoles $P$ follows a power-law of time-to-failure. In addition, the area density of sheared asperity is proportional to the energy release due to the damage evolution (e.g., Nagahama 1993a, b; Nagahama and Yoshii, 1993, 1994), and the summation of energy releases is generally quantified by the cumulative Benioff strain-release $\Omega$ (e.g., Turcotte et al., 2003; Kawada and Nagahama, 2006).

Both electromagnetic and mechanical energy releases prior to the failure or seismic events are caused by the change in area density of sheared asperities (the damage evolution). Finally we note that Muto et al. (2007) reported that the change in area density of asperities promotes frictional discharge (plasma generation) and that the change also causes Benioff electromagnetic radiation.

\section{Discussions}

We have investigated the origins of critical phenomena in electromagnetic and mechanical energy release in terms of damage evolution, and now discuss the relationship between electromagnetic and mechanical energy release processes in terms of irreversible thermodynamics including time-scale invariance.

5.1 Irreversible thermodynamics of damage evolution and electric dipole prior to rock failure or a large earthquake

We now consider irreversible thermodynamics for damage evolution and the electric dipole, defined by the Gibbs free energy per unit volume $G=G(s, \sigma, \alpha, P)$ where $s$ is the entropy density (e.g. de Groot and Mazur, 1962; Nagahama and Teisseyre, 1998; Jou et al., 2001). The total differential of $G$ is given by

$d G=-s d T-\frac{\partial G}{\partial \sigma} d \sigma-\frac{\partial G}{\partial P} d P+\frac{\partial G}{\partial \alpha} d \alpha$,

where $T$ is the absolute temperature, $\partial G / \partial \sigma$ can be considered as the macroscopic elastic strain $\varepsilon$, and $\partial G / \partial P$ as the electric field $\tilde{E}$. When the process is isothermal and linearly irreversible, the phenomenological relationships for $\alpha$ and $P$ can be written as

$\frac{d \alpha}{d t}=-\Gamma \frac{\partial G}{\partial \alpha}$,

$P=\kappa \tilde{E}$,

where $\Gamma$ is a constant reflecting the temporal scale of the irreversible process, and $\kappa$ is the electric susceptibility. Equation (8) describes to the energy release rate associated with damage evolution (e.g. Myasinov et al., 1990; Lemaitre, 1990; Lyakhovsky et al., 1993, 1997; Kawada and Nagahama, 2006) and corresponds to the $J$-integral in fracture mechanics (e.g., Rice, 1968; Lyakhovsky et al., 1993) and Ginzburg-Landau equation (e.g., Honenberg and Halperin, 1977; Lyakhovsky et al., 1993).

We can write Maxwell relations for the last two terms in Eq. (7) as

$$
\left(\frac{\partial M}{\partial P}\right)_{T, \sigma, \alpha}=\left(\frac{\partial \tilde{E}}{\partial \alpha}\right)_{T, \sigma, P},
$$

where $M \equiv \partial G / \partial \alpha$, and the change in $\tilde{E}$ are given as

$\delta P=\left(\frac{\partial M}{\partial \tilde{E}}\right) \delta \alpha$,

wherein $\delta$ represents the incremental change. Here, we redefined $d \alpha / d t$ in Eq. (5) as a separable function,

$\frac{d \alpha}{d t} \propto Z(x, y, z)\left(t_{c}-t\right)^{\frac{2-\rho}{\rho-1}}$,

where $Z(x, y, z)$ relates to the coordinates $(x, y, z)$. Combining Eqs. (9), (11) and (12) leads to

$P=P_{1}-P_{2}\left(t_{c}-t\right)^{\frac{1}{\rho-1}}$,

where $P_{1}$ and $P_{2}$ are constants. From Eqs. (9) and (13), $P$ can be seen to depend on the power-law of time-to-failure (time-to-earthquake). Thus the electric polarization is associated with damage evolution, which affects the Benioff electromagnetic radiation.

In Sect. 4, we confined the damage parameter $\alpha$ to the brittle damage evolution. As defined in Sect. 3, $\alpha$ can be also regarded as the density of the line vacancies or dislocations. The change in electric field due to the motion of charged dislocation can then be described by the irreversible thermodynamics in Eq. (7) (Nagahama and Teisseyre, 1998; 
Teisseyre and Nagahama, 1999, 2001). Thus the irreversible thermodynamics for damage evolution is applicable to mechanisms generating the electromagnetic signals, such as the motion of charged dislocation and the opening and propagation of cracks, and these mechanisms relating the time-scale invariant evolution of damage contribute to Benioff electromagnetic radiation.

5.2 Rock magnetization associated with damage evolution prior to failure

We can also discuss the seismo-magnetic effect associated with damage evolution based on the analogous framework of irreversible thermodynamic in Sect. 5.1. The Gibbs free energy for rock magnetization is defined by Eq. (7) after replacing $P$ by the magnetic field $H$, and then $\partial G / \partial H$ can be regarded as the magnetization $J$ (e.g., de Groot and Mazur, 1962; Nakamura and Nagahama, 1997b, 1999, 2001). In this case, $J$ and $H$ have a linear relation $J=\chi H$, where $\chi$ is the magnetic susceptibility, and the Maxwell relation between $\alpha$ and $H$ yields a relation,

$\delta J=\left(\frac{\partial M}{\partial H}\right) \delta \alpha$,

Then, Eqs. (12) and (14) lead to

$J=J_{1}-J_{2}\left(t_{c}-t\right)^{\gamma_{J}}, \gamma_{J}=\frac{1}{\rho-1}$,

where $J_{1}$ and $J_{2}$ are constants.

Based on these theoretical remarks, we analyze data of remanent magnetization change associated with repetitive creep-rate changes (Fig. 3a, Martin and Wyss, 1975). Sudden changes in stress or strain-rate induce the respective creep curves, and the superposition of the creep behaviours yields a temporal power-law change in inelastic volumetric strain $\varepsilon_{V}$, shown in Fig. 3b. When the damage parameter $\alpha$ is regarded as a normalized parameter on the inelastic volumetric strain $\varepsilon_{V} / \tilde{\varepsilon}_{V}$, where $\tilde{\varepsilon}_{V}$ denotes the inelastic volumetric strain at $t=t_{c}, \alpha$ represents the respective creep behaviours and their superposition, $\rho$ regulates the time-scale invariances in the superposition. This is one of the patterns of the mechanical energy release. The changes in magnetization definitely correspond to the repetitive creeps and their superposition, which supports the theoretical relations in Eqs. (14) and (15) (Fig. 3b). Thus the time-scale invariant change is manifest in remanent magnetization changes due to damage evolution prior to rock failure. Although the seismo-magnetic effect is not often greater than the stable level of geomagnetic effects (e.g., Rikitake, 1976), it is not always trivial to recognize the trend of the temporal power-law change in the magnetization around the fault zone.
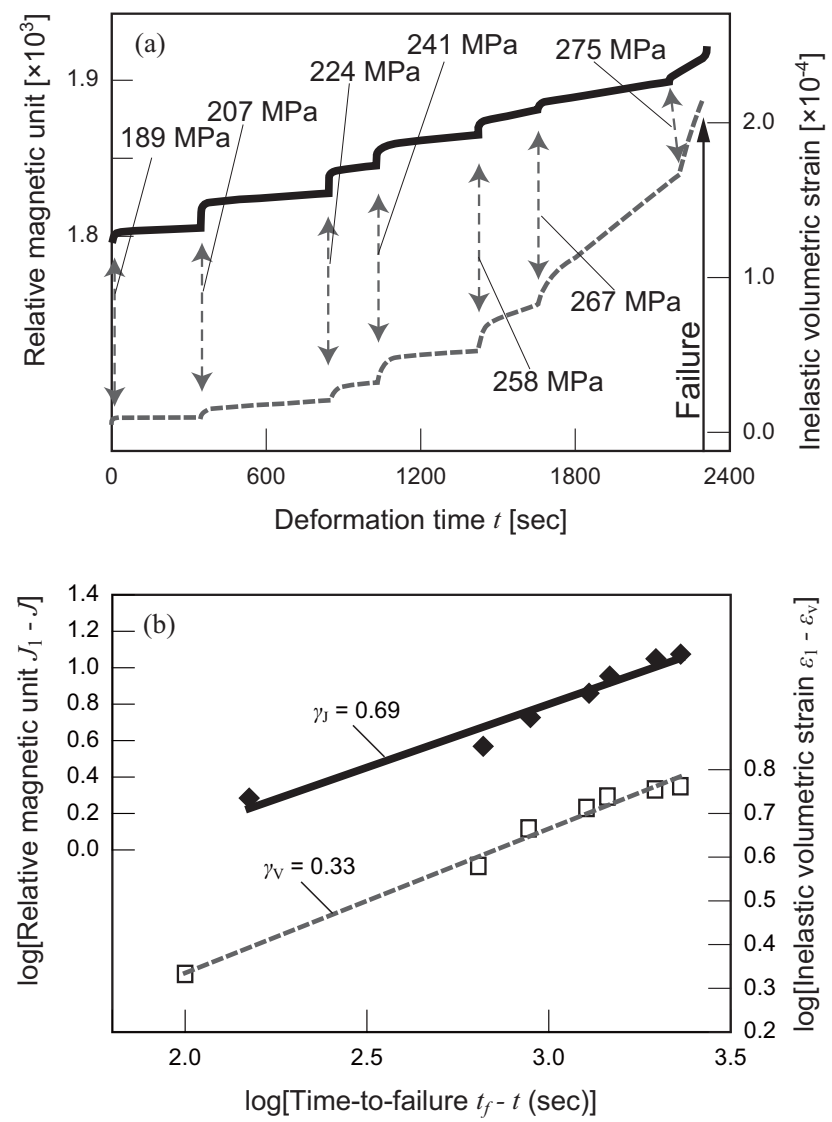

Fig. 3. The temporal changes in remanent magnetization change and inelastic volumetric strain. (a) The temporal evolution of relative magnetic unit associated with repetitive creep-rate changes. The changing points in creep-rate are regarded as seismic events. This figure is adapted from Martin and Wyss (1975). (b) Log$\log$ plots of relative magnetic unit, inelastic volumetric strain $\varepsilon_{V}$ (a type of damage) and time-to-failure. The plots at changing points of creep-rate in Fig. 3a are analyzed. The fitted lines follow the respective temporal power-laws given in Eq. (15) and $\varepsilon_{V}=\varepsilon_{1}-\varepsilon_{2}\left(t_{f}-t\right)^{\gamma_{V}}$, where $\gamma_{V}, \varepsilon_{1}$ and $\varepsilon_{2}$ are constants.

\section{Conclusions}

To explain the time-scale invariances in preseismic electromagnetic and mechanical energy releases, we have investigated the damage mechanics to represent both brittle and plastic processes inducing the electromagnetic radiation. The irreversible thermodynamics with time-scale invariance reveals that the time-scale invariant evolution of damage (such as opening and propagation of cracks, motion of charged dislocation, area-enlargement of sheared asperities, and repetitive creep-rate changes) produces the temporal variation in electromagnetic radiation and mechanical energy releases prior to failure or seismic events, and causes patterns in the Benioff electromagnetic radiation and cumulative Benioff strain-release. Using the irreversible thermodynamics, we 
find also that the time-scale invariant pattern of magnetic energy release due to damage evolution of repetitive creep-rate changes can occur prior to rock failure.

Acknowledgements. The authors thank D. Triantis and an anonymous reviewer for their insightful review comments, and F. Vallianatos for a useful discussion. We are also grateful to J. Greenhough for revising the English style of this manuscript. This study was conducted as a part of the 21 st Century Center-OfExcellence Program, "Advanced Science and Technology Center for the Dynamic Earth", of Tohoku University. The first author (Y. Kawada) was financially supported by the JSPS Research Fellowship for Young Scientists.

Edited by: P. F. Biagi

Reviewed by: D. Triantis and another anonymous referee

\section{References}

Anastasiadis, C., Triantis, D., and Hogarth, C. A.: Comments on the phenomena underlying pressure stimulated currents in dielectric rock materials, J. Mater. Sci., 42, 2538-2542, 2007.

Ben-Zion, Y. and Lyakhovsky, V.: Accelerated seismic release and related aspects of seismicity patterns on earthquake faults, Pure Appl. Geophys., 159, 2385-2412, 2002.

Biot, M. A.: Theory of stress-strain relation in anisotropic viscoelasticity and relaxation phenomena, J. Appl. Phys., 25, 13851391, 1954.

Bowman, D. D, Ouillon, G., Sammis, C. G, Sornette, A., and Sornette, D.: An observational test of the critical earthquake concept, J. Geophys. Res. B, 103, 24 359-24 372, 1998.

Bufe, C. G. and Varnes, D. J.: Predictive modeling of the seismic cycle of the greater San Francisco Bay region, J. Geophys. Res. B, 90, 12 575-12 582, 1993.

Czechowski, Z.: A kinetic model of crack fusion, Geophys. J. Int., 104, 419-422, 1991.

Czechowski, Z.: Dynamics of fracturing and cracks, Theory of Earthquake Premonitory and Fracture Processes, edited by: Teisseyre, R., Polish Scientific Publishers, 447-469, 1995.

de Groot, S. R. and Mazur, P.: Non-equilibrium Thermodynamics, North-Holland, Amsterdam, 1962.

Eftaxias, K., Frangos, P., Kapiris, P., Polygiannakis, J., Kopanas, J., and Peratzakis, A.: Review and a model of pre-seismic electromagnetic emissions in terms of fractal electrodynamics, Fractals, 12, 243-273, 2004.

Eftaxias, K. A., Kapiris, P. G., Balasis, G. T., Peratzakis, A., Karamanos, K., Kopanas, J., Antonopoulos, G., and Nomicos, K. D.: Unified approach to catastrophic events: from the normal state to geological or biological shock in terms of spectral fractal and nonlinear analysis, Nat. Hazards Earth Syst. Sci., 6, 205-228, 2006, http://www.nat-hazards-earth-syst-sci.net/6/205/2006/.

Eftaxias, K., Panin, V. E., and Deryugin, Ye. Ye.: Evolution-EM signals before earthquakes in terms of mesomechanics and complexity, Tectonophysics, 431, 273-300, 2007.

Finkelstein, D., Hill, R. D., and Powell, J. R.: The piezoelectric theory of earthquake lightning, J. Geophys. Res. 78, 992-993, 1973.
Freund, F.: Time-resolved study of charge generation and propagation in igneous rocks, J. Geophys. Res. B, 105, 11 001-11 019, 2000.

Gokhberg, M. B., Mogounov, V. A., and Pokhotelov, A. O.: Earthquake Prediction: Seismo-Electromagnetic Phenomena. Gordon and Breach Publishers, Singapore, 1995.

Honenberg, P. C. and Halperin, B. I.: Theory of dynamic critical phenomena, Rev. Mod. Phys., 49, 435-480, 1977.

Jou, D., Casas-Vázquez, J., and Lebon, G.: Extended Irreversible Thermodynamics, 3rd Ed., Springer-Verlag, Berlin, 2001.

Kapiris, P. G., Balasis, G. T., Kopanas, J. A., Antonopoulos, G. N., Peratzakis, A. S., and Eftaxias, K. A.: Scaling similarities of multiple fracturing of solid materials, Nonlin. Processes Geophys., 11, 137-151, 2004a.

Kapiris, P. G., Eftaxias, K. A., and Chelidze, T. L.: Electromagnetic signature of prefracture criticality in heterogeneous media, Phys. Rev. Lett., 92, 065702, doi:10.1103/PhysRevLett.92.065702, 2004b.

Kawada, Y. and Nagahama, H.: Viscoelastic behaviour and temporal fractal properties of lherzolite and marble, Terra Nova, 16, 128-132, 2004.

Kawada, Y. and Nagahama, H.: Cumulative Benioff strain-release, modified Omori's law and transient behaviour of rocks, Tectonophysics, 424, 157-166, 2006.

Kawada, Y., Nagahama, H., and Hara, H.: Irreversible thermodynamic and viscoelastic model for power-law relaxation and attenuation of rocks, Tectonophysics, 427, 255-263, 2006.

Kawada, Y., Nagahama, H., Omori, Y., Yasuoka, Y., Ishikawa, T., Tokonami, S., and Shinogi, M.: Time-scale invariant changes in atmospheric radon concentration and crustal strain prior to a large earthquake, Nonlin. Processes Geophys., 14, 123-130, 2007 , http://www.nonlin-processes-geophys.net/14/123/2007/.

Kern, J. W.: The effect of stress on the susceptibility and magnetization of a partially magnetized multidomain system, J. Geophys. Res., 66, 3807-3816, 1961.

Lankford, J.: The role of tensile microfracture in the strain rate dependence of compressive strength of fine-grained limestone analogy with strong ceramics, Int. J. Rock Mech. Min. Sci. Geomech. Abstr., 18, 173-175, 1981.

Lemaitre, J.: A Course on Damage Mechanics, Springer, Berlin, 1990.

Lyakhovsky, V., Ben-Zion, Y., and Agnon, A.: Distributed damage, faulting, and friction, J. Geophys. Res. B, 102, 27 635-27 649, 1997.

Lyakhovsky, V., Podladchikov, Y., and Poliakov, A.: A rheological model of a fractured solid, Tectonophysics, 226, 187-198, 1993.

Main, I. G.: Applicability of time-to-failure analysis to accelerated strain before earthquakes and volcanic eruptions, Geophys. J. Int., 139, F1-F6, 1999.

Martin III, R. J. and Wyss, M.: Magnetism of rocks and volumetric strain in uniaxial failure tests, Pure Appl. Geophys., 113, 51-61, 1975.

Mizutani, H., Ishido, T., Yokokura, T., and Ohnishi, S.: Electrokinetic phenomena associated with earthquakes, Geophys. Res. Lett., 3, 365-368, 1976.

Molchanov, O. A. and Hayakawa, M.: Generation of ULF electromagnetic emissions by microfracturing, Geophys. Res. Lett., 22, 3091-3094, 1995. 
Muto, J. and Nagahama, H.: Dielectric anisotropy and deformation of crustal rocks: physical interaction theory and dielectric mylonites, Phys. Earth Planet. Inter., 141, 27-35, 2004.

Muto, J., Nagahama, H., Miura, T., and Arakawa, I.: Frictional discharge at fault asperities: origin of fractal seismoelectromagnetic radiation, Tectonophysics, 431, 113-122, 2007.

Myasnikov, V. P., Lyakhovsky, V. A., and Podladchikov, Y.: Nonlocal model of a viscoelastic body with differential moduli in tension and composition, Sov. Phys. Dokl., 35. 444-445, 1990. (Translated from Dokl. Akad Nauk, SSSR, 312, 302-305, 1990, in Russian.)

Nagahama, H.: Fractal fragment size distribution for brittle rocks, Int. J. Rock Mech. Min. Sci. Geomech. Abstr., 30, 469-471, 1993a.

Nagahama, H.: A fractal criterion for ductile and brittle fracture, J. Appl. Phys., 75, 3220-3222, 1993b.

Nagahama, H. and Teisseyre, R.: Thermodynamics of line defects and transient electric current: electromagnetic field generation in earthquake preparation zone, Acta Geophys. Pol., 46, 35-54, 1998.

Nagahama, H. and Yoshii, K.: Fractal dimension and fracture of brittle rocks, Int. J. Rock Mech. Min. Sci. Geomech. Abstr., 30, 173-175, 1993.

Nagahama, H. and Yoshii, K.: Scaling laws of fragmentation, Fractal and Dynamical Systems in Geosciences, edited by: Kruhl, J. H., Springer-Verlag, Berlin, 25-36, 1994.

Nakamura, N. and Nagahama, H.: Magnetic susceptibility and plastic strain of rocks by the differential geometric theory of the physical interaction field, Phys. Chem. Earth, 22, 167-173, 1997a.

Nakamura, N. and Nagahama, H.: Anisotropy of magnetic susceptibility and plastic strain of rocks - a Finsler geometrical approach -, Acta Geophys. Pol., 45, 333-354, 1997b.

Nakamura, N. and Nagahama, H.: Geomagnetic field perturbation and fault creep motion: a new tectonomagnetic model, Atmospheric and Ionospheric Electromagnetic Phenomena Associated with Earthquakes, edited by: Hayakawa, M., TERRAPUB, Tokyo, 307-323, 1999.

Nakamura, N. and Nagahama, H.: Tectono- and chemicomagnetic effects in tectonically active regions, Earthquake Thermodynamics and Phase Transformations in Earth's Interior, edited by: Teisseyre, R. and Majewski, E., Academic Press, New York, 553-564, 2001.

Nanjo, K. and Nagahama, H.: Fractal properties of spatial distributions of aftershocks and active faults, Chaos, Solitons \& Fractals, 19, 387-397, 2004a.

Nanjo, K. and Nagahama, H.: Discussions on fractals, aftershocks, and active faults: diffusion and seismo-electromagnetism, Arab. J. Sci. Eng., 29, 147-167, 2004b.

Nanjo, K. Z. and Turcotte, D. L.: Damage and rheology in a fibrebundle model, Geophys. J. Int., 162, 859-866, 2005.

Ogawa, T., Oike, K., and Miura, T.: Electromagnetic radiations from rocks, J. Geophys. Res. 90, 6245-6249, 1985.

O'Keefe, S. G. and Thiel, D. V.: A mechanism for the production of electromagnetic radiation during fracture of brittle materials, Phys. Earth Plant. Inter., 89, 127-135, 1995.

Poirier, J. P.: Creep of Crystals, Cambridge Univ. Press, New York, 1985.

Rabinovitch, A., Frid, V., and Bahat, D.: Parameterization of elec- tromagnetic radiation pulses obtained by triaxial fracture of granite samples, Philos. Mag. Lett., 77, 289-293, 1998.

Rabinovitch, A., Frid, V., and Bahat, D.: Gutenberg-Richtertype relation for laboratory fracture-induced electromagnetic radiation, Phys. Rev. E, 65, 011401, doi: 10.1103/PhysRevE.65.011401, 2001.

Rikitake, T.: Earthquake Prediction, Elesevier, Amsterdam, 1976.

Rice, J. R.: A criterion for ductile fracture by the growth of holes, Trans. ASME, J. Appl. Mech., 35, 379-386, 1968.

Rundle, J. B., Klein, W., Turcotte, D. L., and Malamud, B. D.: Precursory seismic activation and critical-point phenomena, Pure Appl. Geophys., 157, 2165-2182, 2000.

Sasai, Y.: Application of the elasticity theory of dislocations to tectonomagnetic modeling, Bull. Earthquake Res. Inst., Univ. Tokyo, 55, 387-447, 1980.

Sasai, Y.: Tectonomagnetic modeling on the basis of the linear piezomagnetic effect, Bull. Earthquake Res. Inst., Univ. Tokyo, 66, 585-722, 1991.

Sasai, Y.: Piezomagnetic fields produced by dislocation surfaces, Surveys Geophys., 15, 363-382, 1994.

Schapery, R. A.: Application of thermodynamics to thermomechanical, fracture, and birefringent phenomena in viscoelastic media, J. Appl. Phys., 35, 1451-1465, 1964.

Schapery, R. A.: On the characterization of nonlinear viscoelastic materials, Polym. Eng. Sci., 9, 295-310, 1969.

Sornette, D.: Critical Phenomena in Natural Sciences, 2nd Ed., Springer, Berlin, 2003.

Stacey, F. D.: The seismomagnetic effect, Pure Appl. Geophys., 58, 5-22, 1964.

Takeuchi, A. and Nagahama, H.: Voltage changes induced by stickslip of granites, Geophys. Res. Lett., 28, 3365-3368, 2001.

Takeuchi, A. and Nagahama, H.: Interpretation of charging on fracture or frictional slip surface of rocks, Phys. Earth Planet. Inter., 130, 3365-3368, 2002a.

Takeuchi, A. and Nagahama, H.: Surface charging mechanism and scaling law related to earthquakes, J. Atmos. Electr., 22, 183190, 2002b.

Takeuchi, A. and Nagahama, H.: Scaling laws between seismoelectric/magnetic fields and earthquake magnitude, Terra Nova, 16, 152-156, 2004.

Takeuchi, A. and Nagahama, H.: Electric dipoles perpendicular to a stick-slip plane: a possible source of seismo-electromagnetic radiation. Phys. Earth Planet. Inter., 155, 208-218, 2006.

Teisseyre, R. and Nagahama, H.: Dislocation models of electric field generation in a seismic source zone. Atmospheric and Ionospheric Electromagnetic Phenomena Associated with Earthquakes, edited by: Hayakawa, M., TERRAPUB, Tokyo, 271285, 1999.

Teisseyre, R. and Nagahama, H.: Electric and electromagnetic fields related to earthquake formation. Earthquake Thermodynamics and Phase Transformations in the Earth's Interior, edited by: Teisseyre, R. and Majewski, E., Academic Press, San Diego, 535-552, 2001.

Triantis, D., Stavrakas, I., Anastasiadis, C., Kyriazopoulos, A., and Vallianatos, F.: An analysis of pressure stimulated currents (PSC), in marble samples under mechanicsl stress, Phys. Chem. Earth, 31, 234-239, 2006.

Turcotte, D. L., Newman, W. I., and Shcherbakov, R.: Micro and macroscopic models of rock failure, Geophys. J. Int., 152, 718- 
728, 2003.

Tzanis, A. and Makropoulos, K.: Did the 7/9/1999 M5.9 Athens earthquake come with a warning?, Natural Hazards, 27, 85-103, 2002.

Tzanis, A. and Vallianatos, F.: A physical model of electrical earthquake precursors due to crack propagation and the motion of charged edge dislocations, Seismo Electromagnetics: Lithosphere-Atmosphere-Ionosphere Coupling, edited by: Hayakawa, M. and Molchanov, O. A., TERRAPUB, Tokyo, 117130, 2002.

Tzanis, A, Vallianatos, F., and Gruszow, S.: Identification and discrimination of transient electrical earthquake precursors: Fact, fiction and some possibilities, Phys. Earth Planet. Inter., 121, 223-248, 2000.

Vallianatos, F., Triantis, D., Tzanis, A., Anastasiadis, C., and Stacrakas, I.: Electric earthquake precursors: from laboratory results to field observations, Phys. Chem. Earth, 29, 339-351, 2004.
Vallianatos, F. and Tzanis, A.: A model for the generation of precursory electric and magnetic fields associated with the deformation rate of the earthquake focus. Atmospheric and Ionospheric Electromagnetic Phenomena Associated with Earthquakes, edited by: Hayakawa, M., TERRAPUB, Tokyo, 287-305, 1999.

Varnes, D. J.: Predicting earthquakes by analyzing accelerating precursory seismic activity, Pure Appl. Geophys., 130, 661-686, 1989.

Varotsos, P. and Alexopoulos, K.: Thermodynamics of point defects and their relation with bulk properties. North Holland, Amsterdam, 1986.

Yasuoka, Y., Igarashi, G., Ishikawa, T., Tokonami, S., and Shinogi, M.: Evidence of precursor phenomena in the Kobe earthquake obtained from atmospheric radon concentration, Appl. Geochem., 21, 1064-1072, 2006. 